\title{
METHOD OF DESIGNING FUZZY SYSTEM FOR PLANNING ROBOT MANIPULATOR REAL-TIME MOVEMENT IN A STATIC ENVIRONMENT
}

\author{
Daiva NEMEIKŠYTĖ, Latvia University of Life Sciences and Technologies, Faculty of Engineering, Address: 5 J. Cakstes blvd., Jelgava \\ LV-3001, Latvia; nemeiksyte.daiva@llu.lv (corresponding author) \\ Vitalijs OSADČUKS, Latvia University of Life Sciences and Technologies, Faculty of Engineering, Address: 5 J. Cakstes blvd., Jelgava \\ LV-3001, Latvia; vitalijs.osadcuks@llu.lv
}

\begin{abstract}
Under rapid development of science and industry the industrial robots and robotic systems are more widely used in the field of industry. The robots and robotic systems help to manage and master new technological processes efficiently and effectively. In this article there was carried out the analysis of the two degree of freedom (DOF) robot arm, which is widely used in practice in order to reveal its functions and to identify its key dynamic parameters using fuzzy logic system. The mathematical model of robot arm dynamics is composed, which includes the mathematical models of the robot arm links twirling motors and the direct positions task model generating the end point of the robot. After the analysis of the robot model the expanded research was performed - after calculating the missing parameters of experimental 2DOF robot arm motors there was created the robot arm model with „V-REP" and "MATLAB Simulink" software packages that allowed to set the control voltages and to observe the changing in time processes of link velocity trajectories. There is calculated designed planar robot end point trajectory deviation from the dynamic trajectory.
\end{abstract}

Keywords: static obstacles, planned track, fuzzy system, planning real-time movement, robot manipulator.

\section{INTRODUCTION}

During expending of production sector, product making procedures becoming more and more difficult robotic systems are also developing in order to satisfy growing economics requirements. There are many special requirements for robotic systems but the demand for robots in industry and manufacturing is constantly increasing i.e. more than $80 \%$ of the world`s robots are used in industry, and especially robotic arms are now adaptable, that are able to perform a variety of quick and exact tasks and developing abilities of mobiles and service robots (Keighobadi, 2018; Shaoming, 2017)

The subject of research in this paper is robots-manipulators with electric executive devices. One of the most promising trends in designing electromechanical object control systems is the use of the theory of variable structure systems with fuzzy logic. When in the fuzzy, the system is not sensitive to the uncertainty of parameters and external disturbances, which allows for stable properties of a closed system. Control algorithms with fuzzy logic system are simple to implement as they do not require a detailed dynamic model or prolonged machine calculations.

Geometric simulation of a robot manipulator in environment with obstacles involves calculation of the shortest distances between the robot's joint and the closest obstacle from the left or the right side during each iteration (Fig.1). At the same time, the shortest distance to the closest obstacle located on the trajectory of robot's joints is analysed as active variable. It must be noted that geometric computer simulations during calculation of the active shortest distance between the manipulator's joint and the nearest obstacle are different from the functioning of a sensing system that provides realistic shortest distance measurement by sensors.

The present article analyses computer simulation of a two-joint robot manipulator that has static obstacles in its operating zone. Obstacle coordinates in the Cartesian coordinate system are provided at each software iteration as a result of measurements made by distance sensors.

Copyright ( 2019 The Authors. Published by Vytautas Magnus University. This is an open-access article distributed under the terms of the Creative Commons Attribution License (CC BY 4.0), which permits unrestricted use, distribution, and reproduction in any medium, provided the original author and source are credited. 


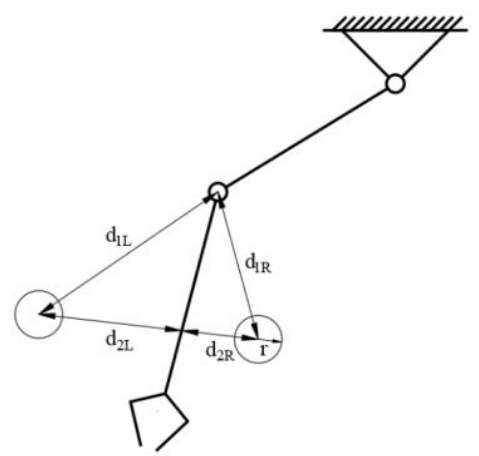

Figure 1. Geometric model of two-joint robot manipulator with active shortest distances between the joints and obstacles

\section{MATERIALS AND METHODS}

The method of designing fuzzy system for planning real-time movement of a robot manipulator in static environment consists of three stages of processing information about the robot and the surrounding environment through designing and functioning of the fuzzy planning system. The use of the method of designing this system is analysed using the example of twojoint robot manipulator (Fig. 2). The validity of the suggested method is based on computer simulation of the system in question.

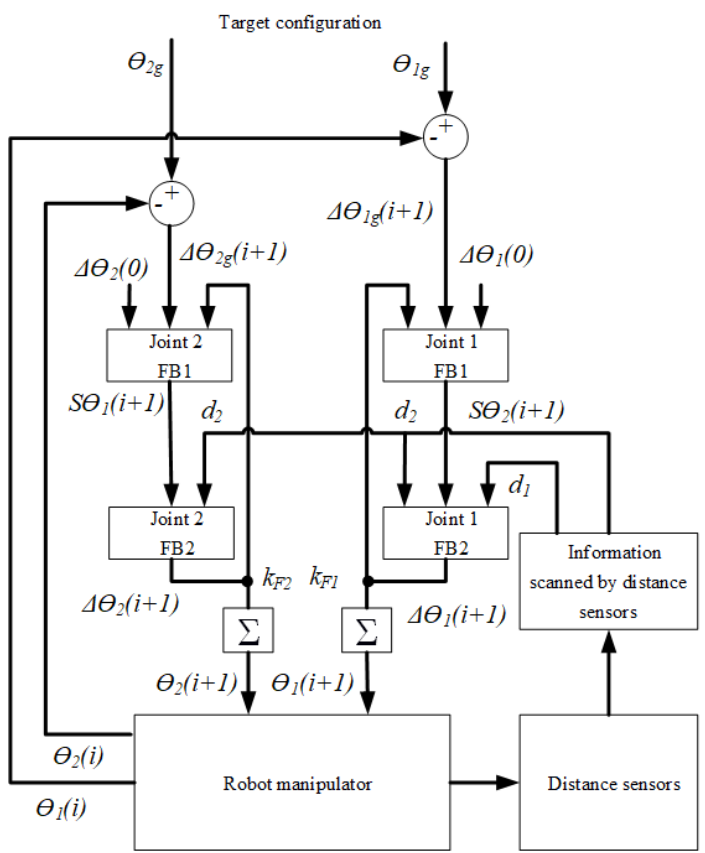

Figure 2. Fuzzy system for planning robot manipulator real-time movement in static environment

During the first stage, the value of the shortest distance between the robot's $n$-joint and the obstacles located in its operating area $\left(d_{n}\right)$ is determined based on the information received from the distance sensors.

During the second stage, the value of robot's $n$-joint initial movement step is calculated $\left(S \Theta_{n}(i+1)\right)$, which is the output of the first fuzzy block (FB1) with the inputs being the values of $n$-joint movement angle change $\left(\Delta \Theta_{n g}(i)\right)$, and also the difference between the target configuration $\left(\Theta_{n g}\right)$ and the current configuration $\left(\Theta_{n}(i)\right)$ of the $n$-joint $\left(\Delta \Theta_{n g}(i+1)=\Theta_{n g}-\Theta_{n}(i)\right)$. It must be noted that the initial value of the system functioning is $\Delta \Theta_{n}(0)=0$. All the output and input parameters of the system can have both positive and negative values.

In the third stage of the method in question, the final change in the manipulator's $n$-joint movement angle $\left(\Delta \Theta_{n}(i+1)\right)$ is determined based on the results of the first and second stages - output of the second fuzzy block (FB2), when the movement angle is determined to be $\Theta_{n}(i+1)=\Theta_{n}(i)+\Delta \Theta_{n}(i+1)$ in a new iteration $(i+1)$ NB2 inputs are $S \Theta_{n}(i+1)$ and $d_{n}$.

Outputs of $\Delta \Theta_{n}$ of a fuzzy system are the positive or negative changes in the movement angle of a manipulator's joint number-one and joint number-two in each new step $(i+1)$. Moreover, there is no need to solve the opposite kinematic task. The values of these outputs are the result of measuring the inputs of a fuzzy system in two planning stages that are provided 
to each joint of the robot's two joints through two fuzzy blocks rather than doing it in one stage of planning which is usually the case in real-time planning systems (Namazov, 2018; Nagesh, 2012; Dersarkissian, 2018)

The principle of the internal feedback implemented in the fuzzy planning system in question will allow for moving through the stage of joint movement $\left(S \Theta_{1}(i+1), S \Theta_{2}(i+1)\right)$ proportional both to the robot's target and true configuration and to the previous value $\left(\Delta \Theta_{I}(i), \Delta \Theta_{2}(i)\right)$. This in turn helps the robot to avoid collision with an unknown obstacle and reach the target.

\section{A. Designing a fuzzy block one of the n-joint}

In the structure of a fuzzy system for planning real-time movement of a two-joint manipulator in static environment two first fuzzy blocks are projected (FB1 of the joint number-one and joint number-two) (Fig. 2).

The first FB1 input of the manipulator's $n$-joint is $\Delta \Theta_{n g}(i+1)$ which is described by four dependence functions (DFs), measured in radians (from $-2 \pi$ to $2 \pi$ ):

1. two trapezium-shaped: FRT (far on the right from the target) and FLT (far on the left from the target);

2. two triangle-shaped: CRT - close on the right from the target; CLT - close on the left from the target (Fig. 3).

Input two $\Delta \Theta_{n}(i)$ is described by two trapezium-shaped dependence functions (SR - step to the right and SL - step to the left) and a triangle-shaped dependence function ( $\mathrm{Z}$ - zero) with the values from $-S_{\max }^{n}$ to $S_{\max }^{n}$, where $S_{\max }^{n}-$ largest step of $n$-joint during each iteration measured in radians (Fig. 3 ).

Output to FB1 $S \Theta_{n}(i+1)$ is described by four dependence functions with the values from $-S_{\max }^{n}$ to $S_{\max }^{n}$. Two trapeziumshaped (SR and SL) and two triangle-shaped (SSR - small step to the right and SSL - small step to the left) (Fig. 3)

Figure 3 depicts the following parameters: $\Delta_{1}=0.6 S_{\max }^{n} ; S_{1}=\Delta_{1} ; S_{2}=0.5 S_{\max }^{n} ; S_{3}=0.3 S_{\max }^{n}$.
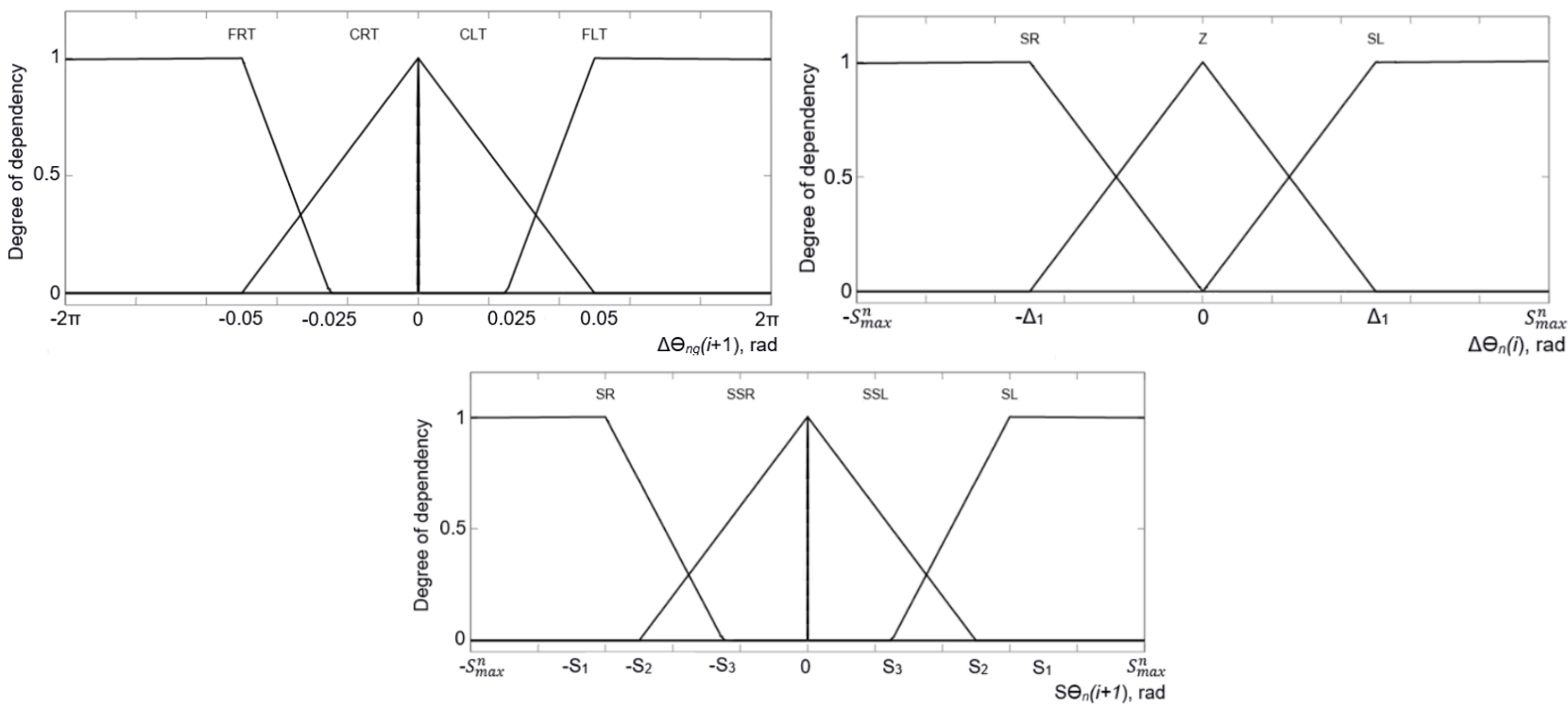

Figure 3. Diagrams of dependence functions of FB1 inputs and outputs of a robot manipulator's $n$-joint

Dependence functions are described by the following formulas (Kong, 2017; Beata, 2018):

$$
\mu_{\text {triangular }}(x ; a, b, c)=\left\{\begin{array}{c}
\frac{x-a}{b-a}, a \leq x \leq b \\
\frac{c-x}{c-b}, b \leq x \leq c \\
0, c \leq x
\end{array}\right\} ; \mu_{\text {trapezoidal }}(x ; a ; b ; c ; d)=\left\{\begin{array}{c}
\frac{x-a}{b-a}, a \leq x \leq b \\
1, b \leq x \leq c \\
\frac{d-x}{d-c}, b \leq x \leq c \\
0, d \leq x
\end{array}\right\}
$$

where $\mu_{\text {triangular }}$ - degree of triangular dependence function; $\mu_{\text {trapezoidal }}$ - degree of trapezoidal dependence function, $x$ - inputs to FB1 $\left(\Delta \Theta_{n g}(i+1)\right.$ or $\left.\Delta \Theta_{n}(i)\right)$ output from FB1 $\left(S \Theta_{n}(i+1)\right)$.

Three-dimensional diagram of fuzzy basic rules and functioning of the manipulator's $n$-joint FB1 is presented in Table 1.

Table 1. Fuzzy basic rules of a $n$-joint with an output parameter $S \Theta_{n}(i+1)$ FB1

\begin{tabular}{|c|c|c|c|c|}
\cline { 2 - 5 } \multicolumn{1}{c|}{} & \multicolumn{4}{c|}{$\Delta \boldsymbol{\Theta}_{n g}(\boldsymbol{i}+\mathbf{1})$} \\
\hline$\Delta \boldsymbol{\Theta}_{\boldsymbol{n g}}(\boldsymbol{i})$ & FRT & CRT & CLT & SSR \\
\hline SR & SR & SSR & SSL & SSR \\
\hline $\mathbf{Z}$ & SSR & SSR & SSL & SSL \\
\hline SL & SSL & SSL & SL \\
\hline
\end{tabular}




\section{B. Designing a fuzzy block two of the n-joint}

In the structure of a fuzzy system for planning real-time movement of a two-joint manipulator in static environment two fuzzy blocks number-two are projected (FB2 of the joint number-one and joint number-two) (Fig. 2).

Input $d_{1}(i+1)$ to the FB2 of the joint number-one is described in four dependence functions with the values from $-d_{1 \max }$ to $d_{1 \max }\left(d_{1 \max }\right.$ is the largest possible value of the distance between the first joint and an obstacle from fuzzy range of values, $\mathrm{m})$ : two triangle-shaped dependence functions ( $\mathrm{CR}$ - close on the right and $\mathrm{CL}$ - close on the left); two trapezoidal dependence functions (FR - far on the right FL - far on the left) (Fig. 4).

Input $S \Theta_{1}(i+1)$ is in turn described by two trapezoidal dependence functions (SR and SL) and a triangular dependence functions (N) with the values from $-S_{\max }^{1}$ to $S_{\max }^{1}\left(S_{\max }^{1}\right.$ is the largest movement step of the joint number-one in each iteration, measured in radians) (Fig. 4).
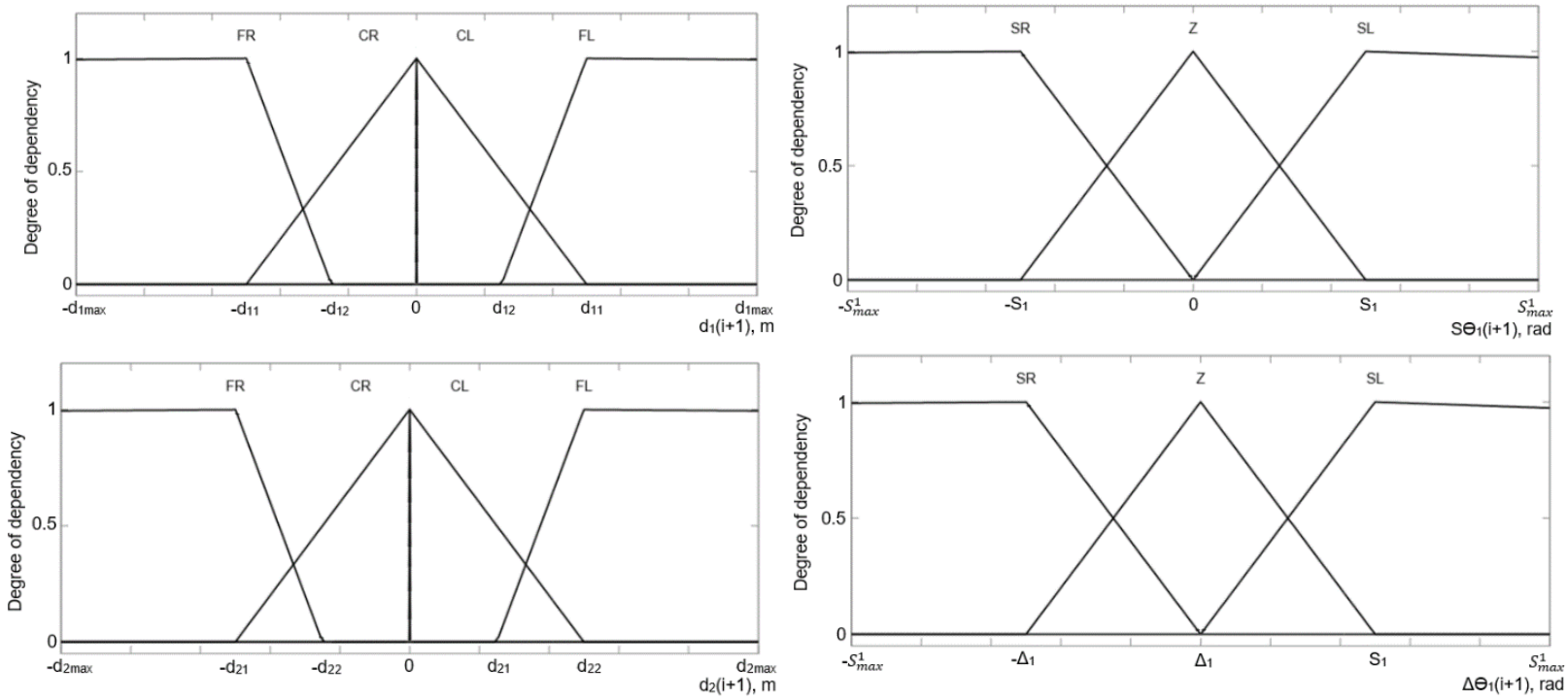

Figure 4. Diagrams of dependence functions of FB2 inputs and outputs of the robot manipulator's joint number-one

Figure 4 depicts the following parameters: $\Delta_{1}=S_{4}=0,6 S_{\max }^{n} ; d_{11}=\frac{d_{1 \max }}{9} ; d_{12}=\frac{d_{1 \max }}{18} ; d_{21}=\frac{d_{1 \max }}{11,25} ; d_{22}=\frac{d_{1 \max }}{22,5}$.

Input $d_{2}(i+1)$ to the FB2 of the joint number-two is described by six dependence functions with the values from $-d_{2 \max }$ to $d_{2 \max }$ : trapezoidal dependence functions - FR and FL; and triangular dependence function: NR - close on the right, NL close on the left, CR, CL (Fig. 5).

Input $S \Theta_{2}(i+1)$, in turn, is described by three dependence functions with the values from $-S_{\max }^{2}$ to $S_{\max }^{2}$ : trapezoidal dependence functions $-\mathrm{SR}$ and SL, triangular dependence function $-\mathrm{Z}$ (Fig. 5).

Output $\Delta \Theta_{2}(i+1)$ from FB2 is described by seven trapezoidal dependence functions with the value range from $-S_{\max }^{2}$ to $S_{\text {max }}^{2}$ : six trapezoidal dependence functions (3SR, 2SR, SR, SL, 2SL, 3SL and triangular dependence function $-\mathrm{Z}$ (Fig. 5).
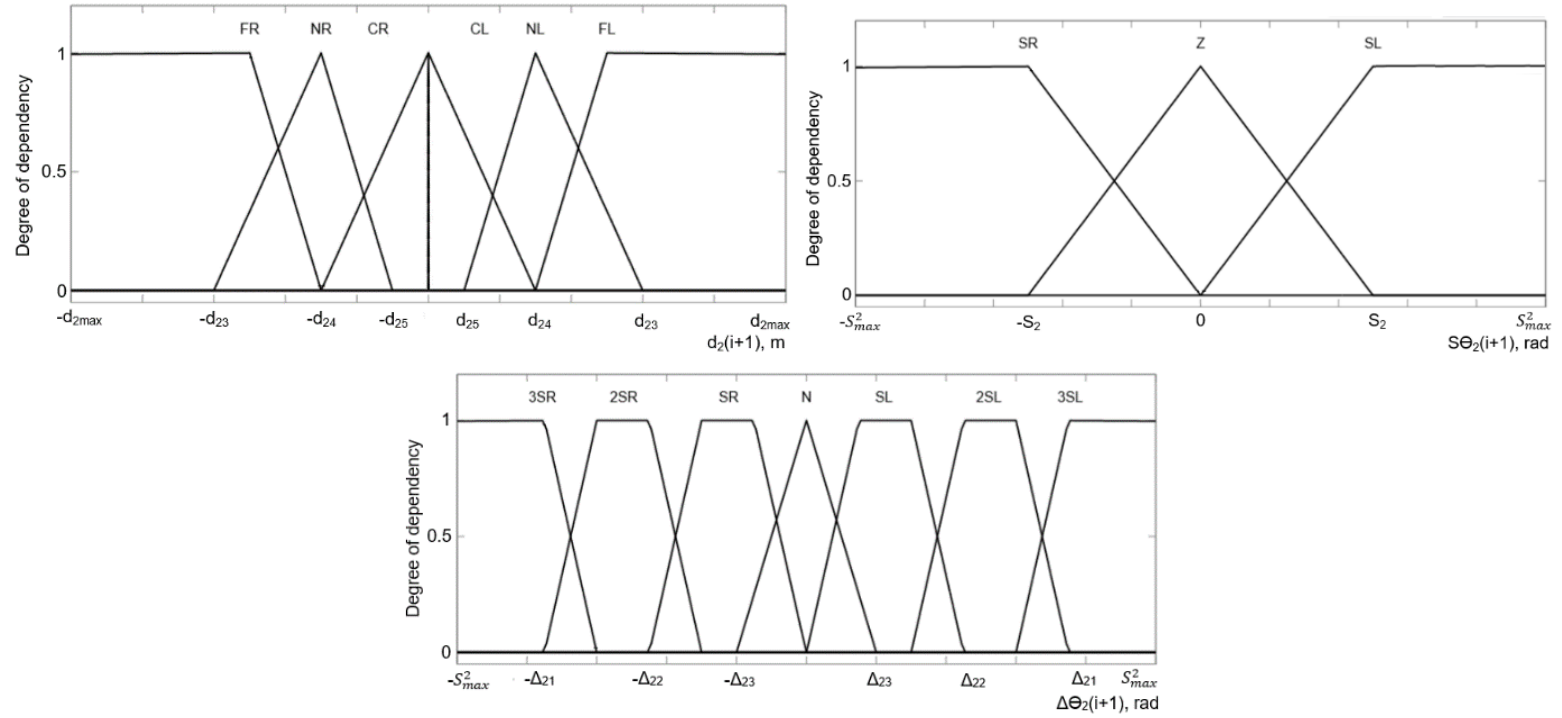

Figure 5. Dependence functions of FB2 inputs and outputs of a robot manipulator's joint number- two 
Figure 5 depicts the following parameters: $d_{23}=\frac{d_{2 \max }}{15} ; d_{24}=\frac{d_{2 \max }}{22,5} ; d_{25}=\frac{d_{2 \max }}{45} ; S_{5}=0.6 S_{\max }^{2} ; \Delta_{21}=0.625 S_{\max }^{2} ; \Delta_{22}=$ $0.375 S_{\max }^{2} ; \Delta_{23}=0.125 S_{\max }^{2}$.

Fuzzy basic rules and a three-dimensional diagram of functioning of the joint number-two FB2 is presented in Table 3.

And finally, input $d_{2}$ is described in four dependence functions with the values from - $\mathrm{d}_{2 \max }$ to $d_{2 \max }\left(d_{2 \max }\right.$ is the largest possible value of the distance between the joint number-two and an obstacle from fuzzy range of values, $\mathrm{m}$ ): two triangleshaped dependence functions - CR and CL); two trapezoidal dependence functions - FR and FL (Fig. 4).

Output from the joint number-one FB2 $\Delta \Theta_{1}(i+1)$ is described by two trapezoidal dependence functions (SR and SL) and a triangular dependence function (Z) with the values from $-S_{\max }^{n}$ to $S_{\max }^{n}$ (Fig. 4).

Basic fuzzy rules of the joint number-one FB2 are presented in Table $2\left(\Delta \Theta_{1}(i+1)-\right.$ output parameter $)$.

Table 2. Basic fuzzy rules of the joint number-one FB2.

\begin{tabular}{|c|c|c|c|c|}
\hline No. & $d_{1}(i+1)$ & $S \Theta_{1}(i+1)$ & $d_{2}(i+1)$ & $\Delta \Theta_{1}(i+1)$ \\
\hline 1 & FR & SR & FR & SR \\
\hline 2 & FR & SR & $\mathrm{CR}$ & $\mathrm{Z}$ \\
\hline 3 & FR & SR & $\mathrm{CL}$ & SR \\
\hline 4 & FR & SR & FL & SR \\
\hline 5 & FR & $\mathrm{Z}$ & FR & $\mathrm{Z}$ \\
\hline 6 & FR & $\mathrm{Z}$ & $\mathrm{CR}$ & $\mathrm{Z}$ \\
\hline 7 & FR & $\mathrm{Z}$ & $\mathrm{CL}$ & $\mathrm{Z}$ \\
\hline 8 & FR & $\bar{Z}$ & FL & $\mathrm{Z}$ \\
\hline 9 & FR & SL & $\mathrm{FR}$ & SL \\
\hline 10 & FR & SL & $\mathrm{CR}$ & SL \\
\hline 11 & FR & SL & $\mathrm{CL}$ & $\mathrm{Z}$ \\
\hline 12 & FR & SL & FL & SL \\
\hline 13 & $\mathrm{CR}$ & SR & FR & SL \\
\hline 14 & $\mathrm{CR}$ & SR & $\mathrm{CR}$ & SL \\
\hline 15 & $\mathrm{CR}$ & SR & CL & $\mathrm{Z}$ \\
\hline 16 & $\mathrm{CR}$ & SR & FL & SL \\
\hline 17 & $\mathrm{CR}$ & $\mathrm{Z}$ & FR & SL \\
\hline 18 & $\mathrm{CR}$ & $\mathrm{Z}$ & $\mathrm{CR}$ & SL \\
\hline 19 & $\mathrm{CR}$ & $\bar{Z}$ & CL & $\mathrm{Z}$ \\
\hline 20 & $\mathrm{CR}$ & $\bar{Z}$ & FL & SL \\
\hline 21 & $\mathrm{CR}$ & SL & FR & SL \\
\hline 22 & $\mathrm{CR}$ & SL & $\mathrm{CR}$ & SL \\
\hline 23 & $\mathrm{CR}$ & SL & CL & $\mathrm{Z}$ \\
\hline 24 & $\mathrm{CR}$ & SL & FL & SL \\
\hline 25 & CL & SR & FR & SR \\
\hline 26 & CL & SR & $\mathrm{CR}$ & $\mathrm{Z}$ \\
\hline 27 & $\mathrm{CL}$ & SR & CL & SR \\
\hline 28 & CL & SR & FL & SR \\
\hline 29 & CL & $\mathrm{Z}$ & FR & SR \\
\hline 30 & $\mathrm{CL}$ & $\bar{Z}$ & $\mathrm{CR}$ & $\mathrm{Z}$ \\
\hline 31 & $\mathrm{CL}$ & $\mathrm{Z}$ & $\mathrm{CL}$ & SR \\
\hline 32 & $\mathrm{CL}$ & $\mathrm{Z}$ & FL & SR \\
\hline 33 & $\mathrm{CL}$ & SL & FR & SR \\
\hline 34 & CL & SL & $\mathrm{CR}$ & $\mathrm{Z}$ \\
\hline 35 & $\mathrm{CL}$ & SL & CL & SR \\
\hline 36 & $\mathrm{CL}$ & SL & FL & SR \\
\hline 37 & FL & SR & FR & SR \\
\hline 38 & FL & SR & $\mathrm{CR}$ & $\mathrm{Z}$ \\
\hline 39 & FL & SR & $\mathrm{CL}$ & SR \\
\hline 40 & FL & SR & FL & SR \\
\hline 41 & FL & $\mathrm{Z}$ & FR & $\mathrm{Z}$ \\
\hline 42 & FL & $\bar{Z}$ & $\mathrm{CR}$ & $\bar{Z}$ \\
\hline 43 & FL & $\mathrm{Z}$ & CL & $\mathrm{Z}$ \\
\hline 44 & FL & $\bar{Z}$ & FL & $\bar{Z}$ \\
\hline 45 & FL & SL & FR & SL \\
\hline 46 & FL & SL & $\mathrm{CR}$ & SL \\
\hline 47 & FL & SL & CL & $\mathrm{Z}$ \\
\hline 48 & FL & SL & FL & SL \\
\hline
\end{tabular}

The fuzzy base rule system used to obtain the required output parameters for each FB is based on the Mamdani algorithm. To calculate the fuzzy intersection (fuzzy AND) within the limits of the established production rules, operations MINIMUM and PRODUCT are used. For example, intersection of fuzzy sets $d_{2}$ and $S \Theta_{2}(i+1)$ (FB2 of the joint number-two) is determined through $\alpha$-rejection (Yangjian, 2018; Sharma, 2016):

$$
\begin{gathered}
\left(d_{2} \cap S \theta_{2}(i+1)\right)_{\alpha}=\left(d_{2}\right)_{\alpha} \cap\left(S \theta_{2}(i+1)_{\alpha}, \forall \alpha \in[0,1] ;\right. \\
\mu_{\rho}\left(d_{2}\right) \cap \mu_{\gamma}\left(S \theta_{2}(i+1)\right)=\mu_{\rho}\left(d_{2}\right) * \mu_{\gamma}\left(S \theta_{2}(i+1)\right) .
\end{gathered}
$$


Table 3. Basic fuzzy rules of the joint number-two FB2 $\left(\Delta \Theta_{2}(i+1)-\right.$ output parameter $)$

\begin{tabular}{|c|c|c|c|c|c|c|}
\hline & \multicolumn{6}{|c|}{$d_{2}(\mathbf{i}+1)$} \\
\hline$S \boldsymbol{\theta}_{2}(i+1)$ & FR & NR & CR & $\mathbf{C L}$ & NL & FL \\
\hline SR & $2 \mathrm{SR}$ & $2 \mathrm{SL}$ & $3 \mathrm{SL}$ & 3SR & 3SR & $3 \mathrm{SR}$ \\
\hline $\mathbf{Z}$ & $\mathrm{Z}$ & SL & $2 \mathrm{SL}$ & 2SR & SR & $\mathrm{Z}$ \\
\hline SL & $3 \mathrm{SL}$ & $3 \mathrm{SL}$ & $3 \mathrm{SL}$ & $3 \mathrm{SR}$ & $2 \mathrm{SR}$ & $2 \mathrm{SL}$ \\
\hline
\end{tabular}

Outputs of the fuzzy blocks are calculated to non-fuzzy using the centre of gravity method (Kong, 2017; Sharma, 2016):

$$
\Delta \theta^{\operatorname{crisp}}=\frac{\sum_{k} b_{k} \int \mu(k)}{\sum_{k} \int \mu(k)}
$$

where $b_{k}$ - dependence functions rule $(\mathrm{k})$ centre; $\int \mu(k)$ - means the area of the dependence functions.

\section{RESULTS AND DISCUSSION}

A two-joint robot manipulator described in Fig. 2 was selected as a research model within the limits of computer simulation for the fuzzy system designed during the dissertation research project for planning robot manipulator real-time movement in static environment. The parameter values of the designed fuzzy blocks for each joint (Fig. 3) are the following:

1. FB1 of the joint number-one: $S_{\text {max }}^{1}=0,01460$ (rad.), $\Delta_{1}=S_{1}=0.008314$ (rad.), $S_{2}=0.006743$ (rad.), $S_{3}=0.003601$ (rad.);

2. FB1 of the joint number-two: $S_{\max }^{2}=0,02031$ (rad.), $\Delta_{1}=\mathrm{S}_{1}=0.01764$ (rad.), $\mathrm{S}_{2}=0.01460$ (rad.), $\mathrm{S}_{3}=0.008314$ (rad.).

Parameter values of FB2 of the joint number-one (Fig. 4) are the following: $\mathrm{d}_{1 \max }=0.8(\mathrm{~m}), \mathrm{d}_{2 \max }=1.24(\mathrm{~m}), \mathrm{d}_{11}=0,1$ $(\mathrm{m}), \mathrm{d}_{12}=0.04(\mathrm{~m}), \Delta_{1}=\mathrm{S}_{4}=0,08314(\mathrm{rad}),. \mathrm{d}_{21}=0.11(\mathrm{~m}), \mathrm{d}_{22}=0.05(\mathrm{~m})$.

Parameter values of FB2 of the joint number-two (Fig. 5) are the following: $\mathrm{d}_{23}=0.08(\mathrm{~m}), \mathrm{d}_{24}=0.05(\mathrm{~m}), \mathrm{d}_{25}=0.02$ (m), $\mathrm{S}_{5}=0.01774$ (rad.), $\Delta_{21}=0.01852$ (rad.), $\Delta_{22}=0.01267$ (rad.), $\Delta_{23}=0.002816$ (rad.).

The values of the obtained fuzzy block dependence functions diagrams (Fig. 3, 4, and 5).

Four static obstacles were placed in the manipulator's operating zone. The shortest distance from a robot's joint and the closest obstacle was calculated using mathematical algorithm simulating the operation of infrared or ultrsound distance sensors.

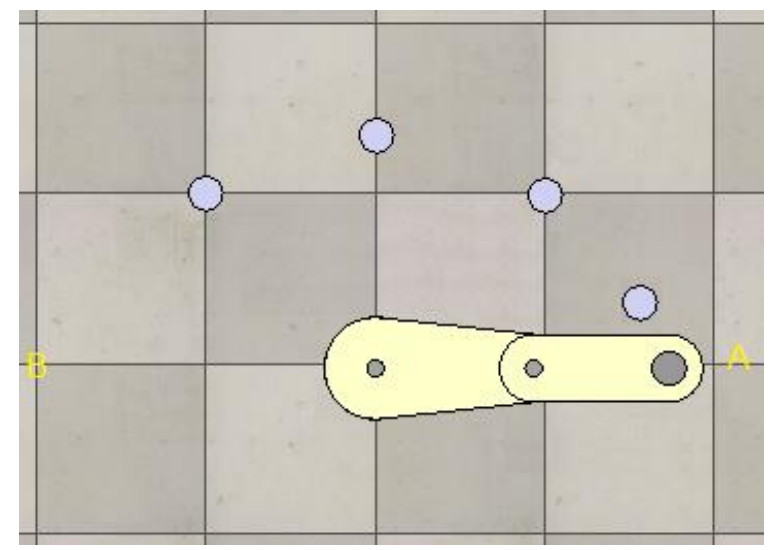

Figure 6. Results of the first test of an intelligent system for planning real-time two-joint robot manipulator movement in static environment based on a fuzzy logic: A - target configuration; B - starting point configuration.

Results of the first test of an intelligent fuzzy logic-based system for planning real-time two-joint robot manipulator movement in static environment containing four static obstacles are presented in Fig. 6. The robot was moving from the start point $\left(\Theta_{1}=0^{0}, \Theta_{2}=0^{\circ}\right)$ to the target point $\left(\Theta_{1}=190^{\circ}, \Theta_{2}=-60^{\circ}\right)$ a certain period of time which was equal to $4.0664 \mathrm{~s}\left(\mathrm{k}_{\mathrm{F} 1}=0.3 ; \mathrm{k}_{\mathrm{F} 2}=\right.$ 0.3). Low amplitude of the robot's joint number-one fluctuations recorded very close to the target configuration, estimated within $\pm 0,15^{0}$. After 2060 software iterations, planning error $\Theta_{1}$ was equal to $0.032107^{0}$, and $\Theta_{2}$ was, respectively, $0.0080534^{0}$.

Figure 7 introduces the results of the second test when the robot was moving from the start point $\left(\Theta_{1}=90^{\circ}, \Theta_{2}=90^{\circ}\right)$ to the target point $\left(\Theta_{1}=180^{\circ}, \Theta_{2}=60^{\circ}\right)$ a certain period of time which was equal to $4.574 \mathrm{~s}\left(\mathrm{k}_{\mathrm{F} 1}=0.3 ; \mathrm{k}_{\mathrm{F} 2}=0.3\right)$. Error of reaching the target (planning error) $\Theta_{2}$ after 2163 software iterations was equal to $0.116464^{0}$. $\Theta_{1}$ error was practically absent as movement of the joint number-one was successfully stopped before possible collusion with the obstacle number-four. In such situation, the robot reached the final configuration. Fig. 8, 9, 10 and 11 presents the diagrams of the following test number-one and test number-two parameters: $\mathrm{d}_{1}, \mathrm{~d}_{2}, \mathrm{~S}_{1}, \mathrm{~S} \Theta_{2}, \Delta \Theta_{1}, \Delta \Theta_{2}, \Theta_{1}$ and $\Theta_{2}$. The manipulator passed beside four obstacles without any collisions. At the same time, a relatively smooth trajectory of its movement was recorded. The second 
test of the system, as evidenced by the above results, was more complicated as it involved movement of robot's joint between two obstacles located within a short distance of each other.

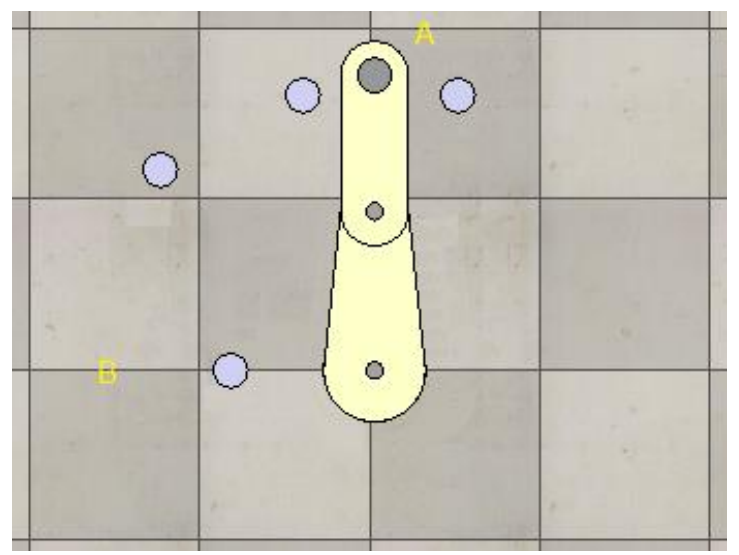

Figure 7. Results of the second test of a fuzzy planning system for two-joint robot manipulator real-time movement in static environment: $\mathrm{A}-$ target configuration; B - starting point configuration.
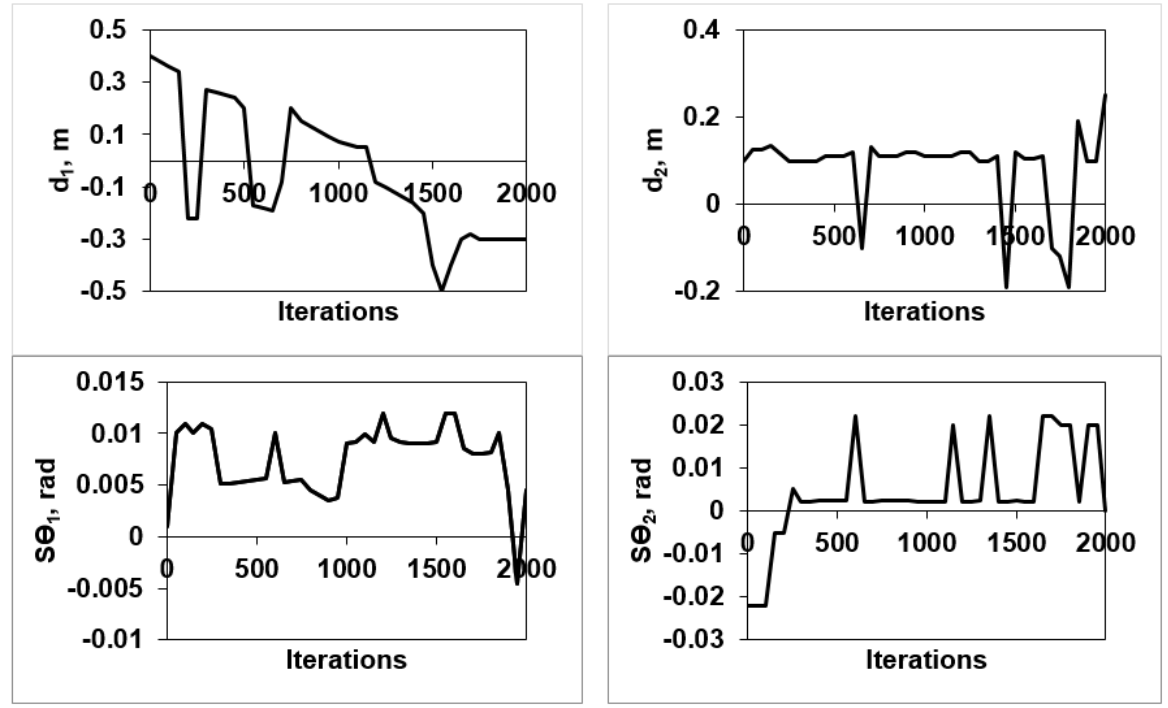

Figure 8. Result parameters of the first test of the fuzzy system
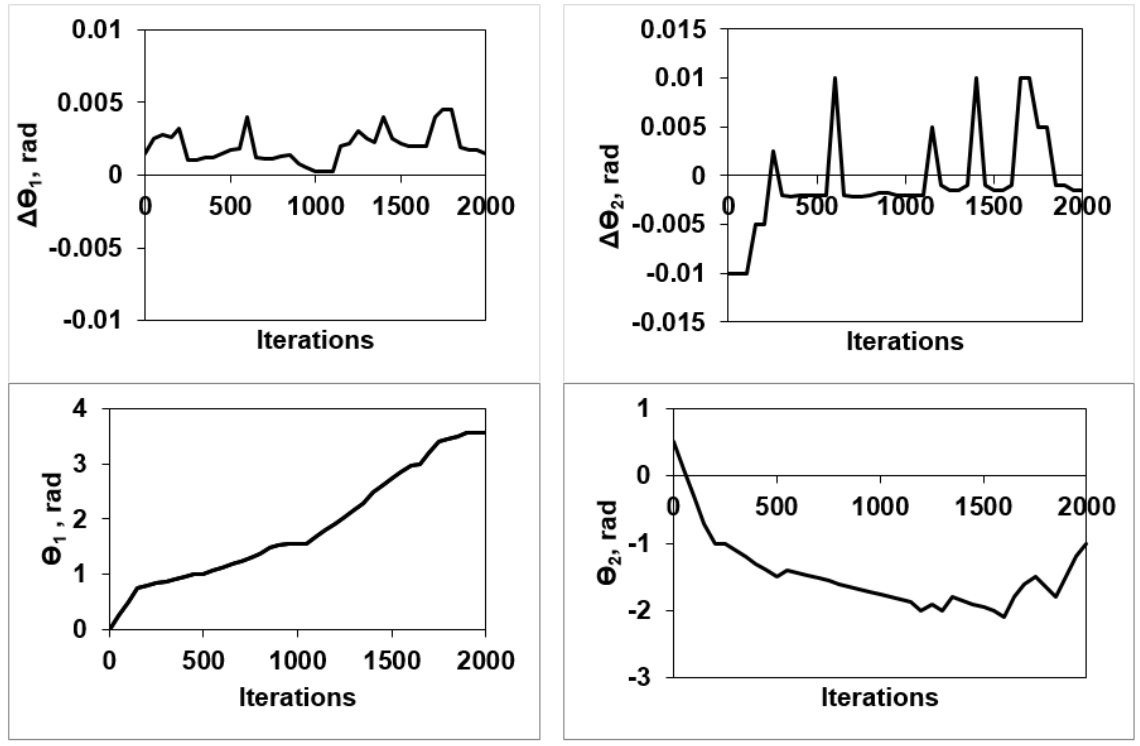

Figure 9. Result parameters of the first test of the fuzzy system 

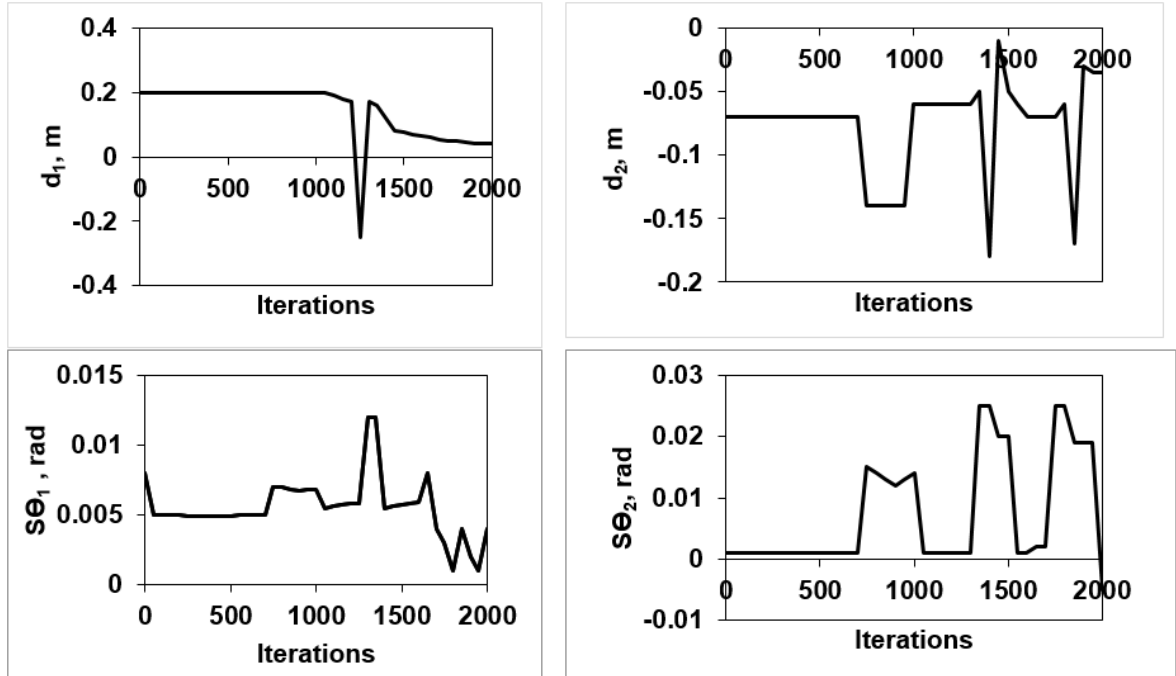

Figure 10. Result parameters of the second test of the fuzzy system
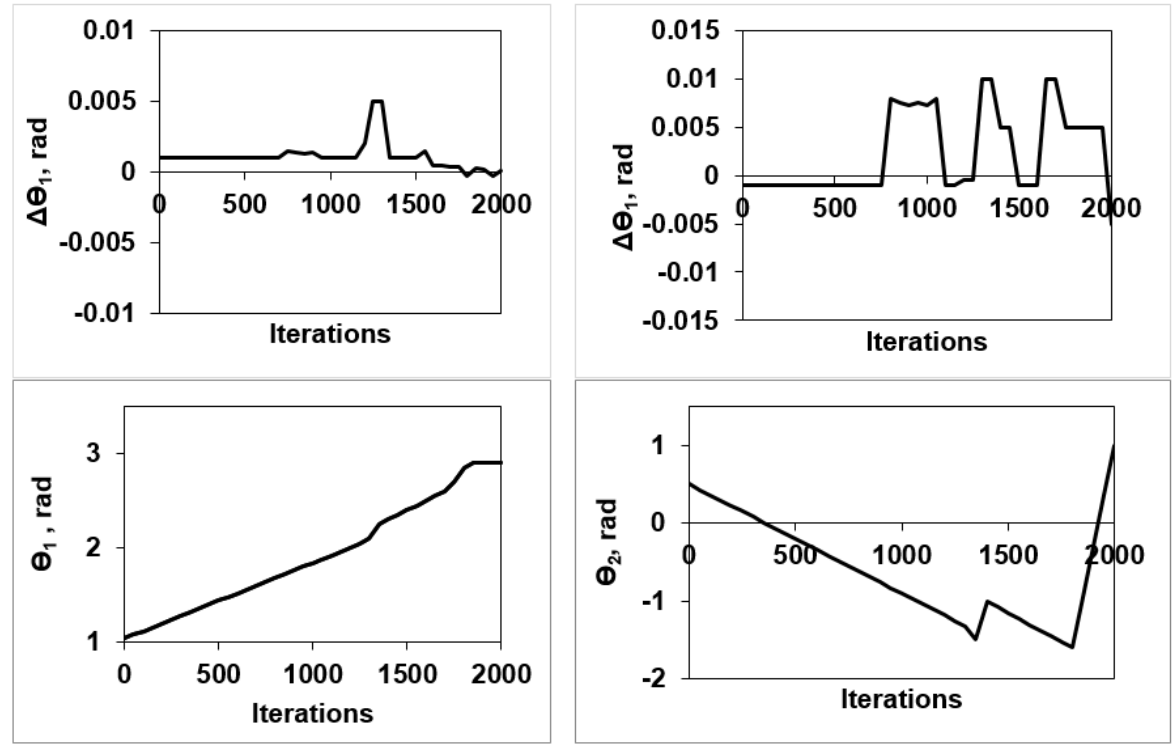

Figure 11. Result parameters of the second test of the fuzzy system

\section{CONCLUSIONS}

During robot manipulator's movement in environment, it simultaneously deals with two tasks: bypassing obstacles and reaching the target point. Therefore, within the scope of the method of designing a fuzzy system operating in real-time for planning robot manipulator's movement in static environment, a fuzzy structure have been suggested consisting of two fuzzy blocks of each joint with the first block calculating the preliminary step value of the robot movement towards the target point depending on the difference between the target and current configuration parameters, and on the value of the preceding step; whereas the second block deals with the problem of not colliding with static obstacles located in its operating zone. The preliminary step and the shortest distance between the robot's joints and the closest obstacles are analysed as the input parameters of the second fuzzy block. Output of the second fuzzy block is the final value of robot's joint step change. Computer simulation has revealed that the largest planning error was $0,15^{0}$ with the oscillatory movements of the robot's joint numbertwo recorded in the target point area.

If it is not possible to find a real-time planning solution for robot manipulator movement in environment with the use of an intelligent system, it is suggested to use a three-dimensional robot instead of a two-joint robot.

\section{REFERENCES}

1. Beata J. 2018. Fuzzy logic controller for robot manipulator control system. Applications of Electromagnetics in Modern Techniques and Medicine (PTZE), Racławice, China, pp. 77-80. https://doi.org/10.1109/PTZE.2018.8503205 
2. Dersarkissian N., Jia R., Feitosa, D. L. 2018. Control of a Two-link Robotic Arm using Fuzzy Logic. IEEE International Conference on "Information and Automation (ICIA)", Wuyishan, China, pp. 481-486. https://doi.org/10.1109/ICInfA.2018.8812444

3. Yangjian W., Xiaofei C. 2018. Manipulator of Robust Fuzzy Adaptive Compensation Control. 3rd Asia-Pacific Conference on Intelligent Robot Systems (ACIRS), Singapore, pp. 61-65. https://doi.org/10.1109/ACIRS.2018.8467251

4. Keighobadi J., Fateh M. M., Chenarani, H. 2018. Adaptive fuzzy passivation control based on backstepping method for electrically driven robotic manipulators. 6th RSI International Conference on "Robotics and Mechatronics" (IcRoM), Tehran, Iran, pp. $292-297$. https://doi.org/10.1109/ICRoM.2018.8657516

5. Kong L. He Dong W. Y., Yang C., Li G. 2017. Bounded control of robotic manipulators via fuzzy logic systems. 36th Chinese Control Conference (CCC), Dalian, pp.140-144. https://doi.org/10.23919/ChiCC.2017.8027334

6. Nagesh, S.B., Lendek, Z., Khalate A.A. and Babuška, R. 2012. Adaptive fuzzy observer and robust controller for a 2-DOF robot arm. 2012 IEEE International Conference on "Fuzzy Systems", Brisbane, QLD, 1-7. https://doi.org/10.1109/FUZZ$\underline{\text { IEEE.2012.6250787 }}$

7. Namazov M. 2018. Fuzzy Logic Control Design for 2-Link Robot Manipulator in MATLAB/Simulink via Robotics Toolbox. Global Smart Industry Conference (GloSIC), Chelyabinsk, pp. 1-5. https://doi.org/10.1109/GloSIC.2018.8570085

8. Shaoming L., Ruipeng L. 2017. Research on trajectory tracking control of multiple degree of freedom manipulator. 32nd Youth Academic Annual Conference of "Chinese Association of Automation" (YAC), Hefei, China, pp. $218-222$. https://doi.org/10.1109/YAC.2017.7967408

9. Sharma R., Gaur P., Mittal A.P. 2016. An enhanced hybrid fuzzy logic controller for robotic manipulator. 3rd International Conference on Computing for Sustainable Global Development (INDIACom), New Delhi, India, pp. 2465-2470. 